\title{
Industrial Experience with the Migration of Legacy Models using a DSL
}

\author{
Mathijs Schuts \\ Philips \\ Best \\ The Netherlands \\ mathijs.schuts@philips.com
}

\author{
Jozef Hooman \\ ESI \& Radboud University \\ Eindhoven \& Nijmegen \\ The Netherlands \\ jozef.hooman@tno.nl
}

\author{
Paul Tielemans \\ Philips \\ Best \\ The Netherlands \\ paul.tielemans@philips.com
}

\begin{abstract}
Software departments of companies that exist for several decades often have to deal with legacy models. Important business assets have been modelled with tools that are no longer preferred within the company. Manually remodelling these models with a new tool would be too costly. In this paper, we describe an approach to migrate from Rhapsody models to models of another tool. To perform the migration, we created a Domain Specific Language (DSL) that accepts Rhapsody models as instances. A generator of this DSL can then produces model instances for the new tool. To get confidence in the transformation in a pragmatic way, we applied a combination of model learning and equivalence checking. Learning has been applied to both the source code generated by Rhapsody and the code generated by the new tool. The resulting models are compared using equivalence checking.

CCS Concepts - Software and its engineering $\rightarrow$ Domain specific languages; State systems; Model-driven software engineering; • Computer systems organization $\rightarrow$ Maintainability and maintenance;
\end{abstract}

Keywords Model-based development, Domain specific languages, Model transformation, Legacy, Tool migration

\section{ACM Reference Format:}

Mathijs Schuts, Jozef Hooman, and Paul Tielemans. 2018. Industrial Experience with the Migration of Legacy Models using a DSL. In RWDSL2018: Real World Domain Specific Languages Workshop 2018, February 24, 2018, Vienna, Austria. ACM, New York, NY, USA, 10 pages. https://doi.org/10.1145/3183895.3183897

Permission to make digital or hard copies of all or part of this work for personal or classroom use is granted without fee provided that copies are not made or distributed for profit or commercial advantage and that copies bear this notice and the full citation on the first page. Copyrights for components of this work owned by others than ACM must be honored. Abstracting with credit is permitted. To copy otherwise, or republish, to post on servers or to redistribute to lists, requires prior specific permission and/or a fee. Request permissions from permissions@acm.org.

RWDSL2018, February 24, 2018, Vienna, Austria

(c) 2018 Association for Computing Machinery.

ACM ISBN 978-1-4503-6355-6/18/02 . .\$15.00

https://doi.org/10.1145/3183895.3183897

\section{Introduction}

High-tech systems are developed over the course of many decades. The complex software created for these systems is partly manually crafted and partly modelled using modelling tools. In the course of time, some of these modelling tools may no longer be preferred. Because the value created with these tools was an investment of many man-years, it is costly and unattractive to manually reimplement the same behaviour using a newer tool. Instead, we describe one project to automatically migrate models from one tool to another by means of a Domain Specific Language (DSL).

This paper addresses the transformation of Rhapsody [11] models at Philips. Rhapsody is a UML-based modelling tool that has been used to develop software components. In Rhapsody, state machines can be modelled graphically. From these models, the Rhapsody tooling generates source code in C++ which can be integrated into the product. The generated source code depends on run-time libraries from Rhapsody that are required to execute the state machine code.

Unfortunately, Rhapsody did not bring the expected benefits. Partly this is due to a wrong use of the tool, but it is also not very convenient to use the Rhapsody tooling in the company's standard way of working. Code, e.g., to describe actions on transitions, has to be entered via a small user interface window and it is difficult to get a good overview of the source code. The main problem is the difficulty to merge code between product lines. Every product line has its development stream in the code repository. Merge errors can easily lead to faults in the resulting product.

This led to the wish to remove the dependency on Rhapsody and to migrate Rhapsody models to Dezyne models. Dezyne is a commercial modelling tool created by the company Verum ${ }^{1}$. With Dezyne, data-independent control components can be modelled using a DSL. This is a so-called horizontal DSL as defined by [18] since it is meant for a specific problem domain, namely data-independent control components. Dezyne models can be formally verified for a fixed set of properties, such as live-lock freedom, dead-lock freedom, determinism and interface conformance. Moreover, source code can be generated from Dezyne models. Dezyne is already used in the company, hence a transformation of

\footnotetext{
${ }^{1}$ https://www.verum.com/
} 
Rhapsody to Dezyne would also reduce the number of tools that have to be supported.

As an intermediate step in the transformation, we use a DSL called Component Modelling \& Analysis (ComMA) [14]. ComMA is a horizontal DSL created at Philips and a company standard to describe state machines. From a ComMA model we generate a Dezyne model. In this way, we avoid a strong dependency on the Dezyne tooling when further developing the migrated components. In addition, it creates the possibility to generate source code from ComMA models in the future.

In general, assume given two modeling languages $\mathrm{A}$ and $\mathrm{B}$, each equipped with a code generator. Then our approach to transform models of language A into models of language B consists of five main steps:

1. Create a DSL that accepts instances of language A.

2. Define a generator which transforms instances of language $\mathrm{A}$ into instances of language $\mathrm{B}$.

3. Generate code for an instance of language $A$ and for its transformed instance of language B.

4. Apply model learning techniques [25] to learn models for both pieces of generated code.

5. Use model checking techniques to verify that the learned models are equivalent. If not, improve the transformation of step 2 .

In the application at Philips described here, we created a DSL that accepts instances of Rhapsody. The generator of the DSL can generate a ComMA model and from the ComMA model a Dezyne model is generated. The main challenges of the transformation are:

- Differences in the syntax of the languages. For instance, the possibilities for connector states and sub states of Rhapsody are not allowed by Dezyne. On the other hand, in Dezyne models all transitions should have an explicit activation which is not required in Rhapsody models.

- Differences in the semantics of the languages. Although there are publications about the semantics of an earlier version of Rhapsody [8] and the ASD approach behind Dezyne [9], the precise semantics of the code generators of both tools is not available.

Given these challenges, model learning and equivalence checking (steps 4 and 5 of our approach) are pragmatic ways to increase the confidence in the transformation.

Another challenge is to apply the approach in an industrial context where it is important to investigate the business case for the application of these techniques. Hence, we calculated the Return On Investment (ROI) for our approach.

This paper is organized as follows. We provide an overview of related work in Section 2. In Section 3 the approach to refactoring a legacy implementation using a DSL is explained. The model transformations which are implemented by a generator of this DSL are described in Section 4. Section 5 explains how confidence in the transformations has been obtained. Concluding remarks are given in Section 6.

\section{Related Work}

The research agenda of [26] mentions that migration from one model-based development tool to another, for instance, using model to model transformations, is required for system evolution. [15] discuss definitions of model transformation techniques. Model to model transformation techniques are described and compared in [3].

To facilitate the migration from one model-based development tool to another, the Object Management Group (OMG) has defined and specified a modelling transformation language: Query Views Transformations (QVT) [17]. According to [22], many UML modelling tools provide the possibility to import and export models in a XML-based language called XML Metadata Interchange (XMI). In the XMI format these models can be transformed from one modelling tool to another using existing XML manipulation tools, e.g., the Extensible Stylesheet Language Transformation (XSLT) tool. [3] describe that XSLT has scalability issues and that [24] has overcome this shortcoming by creating a DSL for applying model transformations. Another approach to replace XSLT is the UML Model Transformation (UMT) tool. [7] describe the empirical successes of UMT by means of a number of transformations, e.g., from UML to J2EE and XDoclet, WSDL-to-UML, UML-to-WSDL and UML-to-BPEL4WS.

[16] describe an industrial project at Philips in which legacy XML files, used for describing field service procedures, are semi-automatically rejuvenated into DSL model instances. These rejuvenated DSL models are transformed into redesigned DSL models from which new $\mathrm{C}++$ source code is generated.

The previously described model transformation technologies assume that the models to be transformed are in XML format. However, Rhapsody stores the models in a proprietary format. Related to our work is an approach that has been applied at the company ASML for a language called Logical Action Component Environment (LACE). LACE defines how different sub-systems are allowed to interact with each other. [23] describe how LACE models are transformed using DSL technology into Event-B models to gain access to multiple specification analysis tools.

[20] present an industrial case study at General Motors where legacy models are converted to AUTomotive Open System ARchitecture (AUTOSAR) models. For the model to model transformations, the MDWorkbench together with the Atlas Transformation Language (ATL) [13] was used [21]. ATL is a QVT-like transformation language [12].

\section{Transformation Approach}

An overview of our transformation is depicted in Figure 1. An example of the textual representation of a Rhapsody model is 


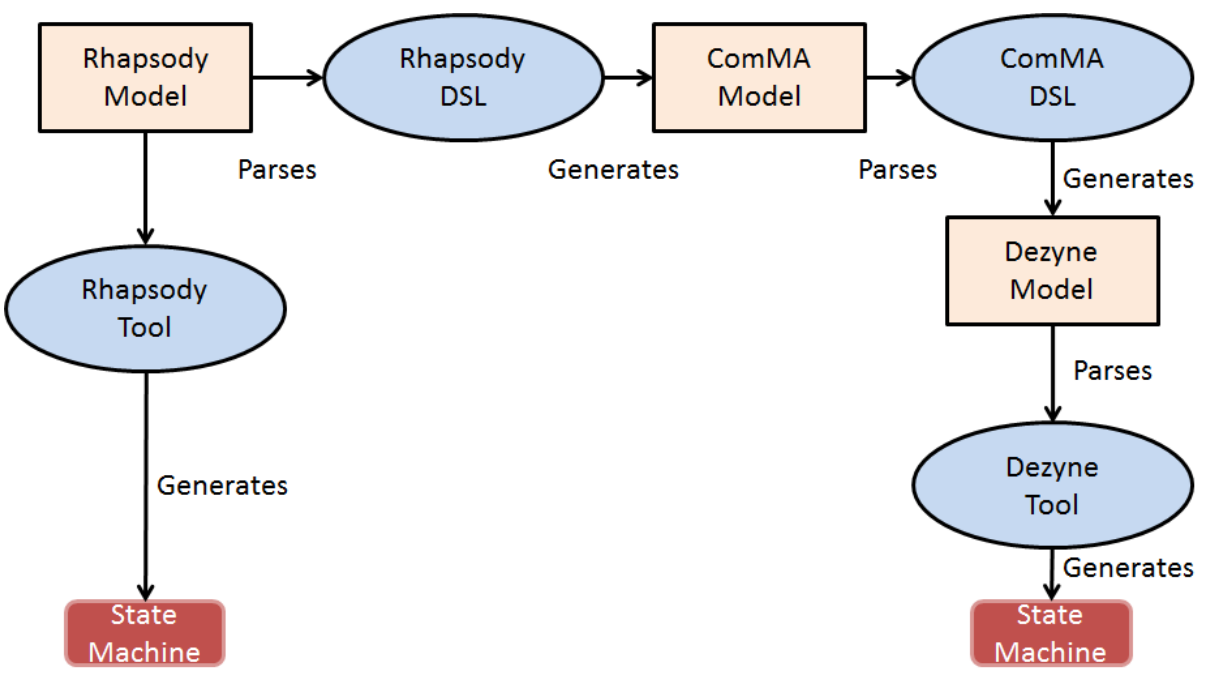

Figure 1. Transformation Approach

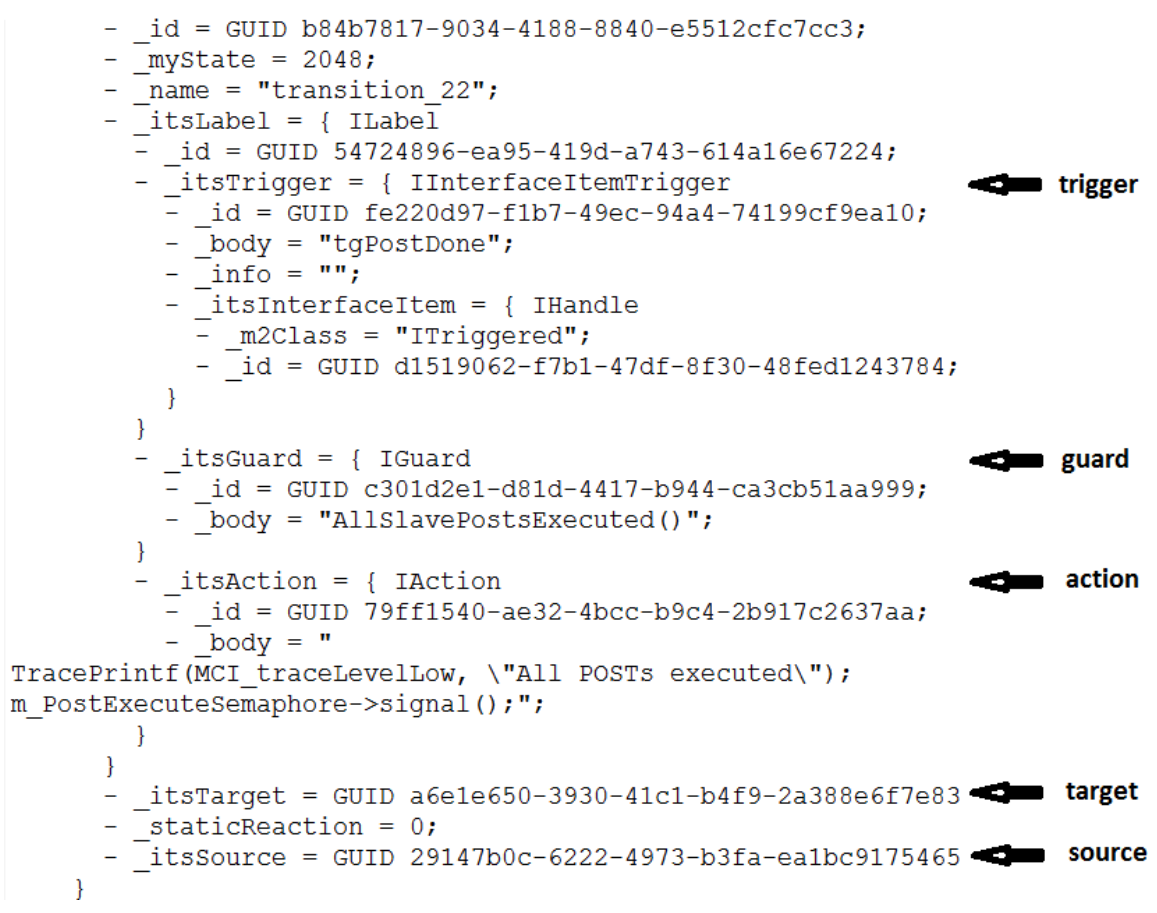

Figure 2. Fragment of a Rhapsody Model

shown in Figure 2. It describes a transition from one state (indicated by itsSource at the end of the figure) to another state (indicated by_itsTarget). The transition is triggered by tgPostDone. When triggered and the guard (AllSlavePostExecuted) evaluates to true, the action body is executed.

We created a DSL, called RhapsodyDSL, which accepts such Rhapsody models as language instances. Eclipse [27] and the Xtext and Xtend plug-ins [1] are used to create this DSL. A generator of this DSL transforms language instances into a ComMA instance. This transformation is described formally in Section 4. Next we extended the framework for the ComMA DSL with a generator that leads to Dezyne models, as described in Section 4.4. Finally, the Dezyne tooling is used to generate a state machine implementation in $\mathrm{C}++$. This replaces the Rhapsody generated state machine in the code base.

\section{State Machine Transformations}

In this section, we describe the essence of the transformation from Rhapsody state machines to ComMA state machines. 
The aim is to define this transformation in such a way that the generation from ComMA to Dezyne is trivial. In Section 4.1 we define the main state machine concepts. The transformation from Rhapsody to ComMA is described in Section 4.2. The main ideas of encoding this in a generator of the RhapsodyDSL can be found in Section 4.3. Section 4.4 illustrates the straightforward translation of ComMA models to Dezyne models.

\subsection{State Machine Definitions}

Our notations are inspired by $[4,6]$.

State machine representation A state machine (SM) is represented using a tuple $<s_{d}, S, T, S u b>$ where

- $S$ is a set of states. A state $s$ has a name, denoted by name $(s)$. Optionally, a state may have a Global Unique IDentifier (GUID), denoted by $G U I D(s)$. The set $S$ may contain so-called connector states (denoted by $\left.s_{c}, s_{c_{1}}, \ldots\right)$. A connector state is a state that can be used to model choices on transitions.

- $s_{d} \in S$ is the default state, also called the initial state, and denoted by default(SM).

- $T$ is a set of transitions. A transition has the from $s_{0} \stackrel{[g] a c t / a}{\longrightarrow} s_{1}$ where

$-s_{0} \in S$ is the source state.

- $g$ is a guard, which is a boolean expression consisting of method calls that evaluate to either true or false. The guard is optional, i.e., can be omitted.

- act is an activation which can be a trigger which is a synchronous method or an asynchronous event. For a synchronous method, the caller has to wait until the callee is available. Events are queued. The activation part is optional.

- $a$ is a list of actions. An action is a method call (which might generate an event). The action part is optional.

$-s_{1} \in S$ is the target state.

For such a transition $t$ we use $\operatorname{source}(t)=s_{0}, \operatorname{guard}(t)=$ $g, \operatorname{activation}(t)=\operatorname{act}, \operatorname{action}(t)=a$, and $\operatorname{target}(t)=$ $s_{1}$.

- $S u b$ is set of sub state machines. A sub state machine is a tuple of the form $<s_{i}, S M_{i}>$ where $s_{i} \in S$ and $S M_{i}$ is a state machine. Note that $S u b$ may be empty.

The states $s_{i}$ occurring in sub state machines are called super states. Super $(s)$ is true iff $s$ is a super state.

Example Figure 3 depicts an example state machine (SM) $<s_{0}, S, T, S u b>$ where

- The set of states $\mathrm{S}$ is $\left\{s_{0}, s_{1}, s_{2}, s_{3}, s_{4}\right\}$. State $s_{2}$ is a connector state.

- The set of transitions $\mathrm{T}$ is $\left\{t_{0}, t_{1}, t_{2}, t_{3}\right\}$ where

$-t_{0}$ is the transition $s_{0} \stackrel{\left[g_{0}\right] a c t_{0} / a_{0}}{\longrightarrow} s_{1}$

$-t_{1}$ is the transition $s_{1} \stackrel{[] a c t_{1} /-}{\longrightarrow} s_{2}$

$-t_{2}$ is the transition $s_{2} \stackrel{\left[g_{1}\right]-/ a_{1}}{\longrightarrow} s_{3}$ $-t_{3}$ is the transition $s_{2} \stackrel{\left[g_{2}\right]-/ a_{2}}{\longrightarrow} s_{4}$

- $\mathrm{Sub}$ is $<s_{4}, S M_{4}>$.

State machine $S M$ contains a sub-state machine $S M_{4}$ which is defined by the tuple $<s_{5}, S_{4}, T_{4}, S u b_{4}>$ where

- The set of states $S_{4}=\left\{s_{5}, s_{6}\right\}$.

- The set of transitions $T_{4}=\left\{s_{5} \stackrel{\left[g_{3} \wedge g_{4}\right] a c t_{2} / a_{3}, a_{4}}{\longrightarrow} s_{6}\right\}$.

- $S u b_{4}=\varnothing$.

Rhapsody models The Rhapsody models considered for the transformations discussed in this paper are state machines, but they satisfy a number of constraints. First of all, states and GUIDs are unique:

- Each state occurs at most once in the hierarchy of states. The same holds for GUIDs. However, there can be multiple states with the same name.

For our transformation, we assume that state names are not post-fixed with a number, which holds for the models to be transformed.

For each transition $t$ we have the following constraints:

- If $t$ has an action list, then the list has length 1.

- If $t$ has a guard, then this is a single method call which returns a boolean value.

- If $\operatorname{source}(t)$ is a connector state then $t$ has no activation. It may have a guard and may have an action. Moreover, target $(t) \neq$ source $(t)$, i.e., connector states do not have self transitions.

- If $\operatorname{source}(t)$ is not a connector state and target $(t)$ is a connector state, then $t$ has an activation, but no guard and no action.

- For each connector state $s_{c}$ there is one incoming transition (for which $s_{c}$ is the target) and two outgoing transitions (for which $s_{c}$ is the source). Moreover, the guard of one outgoing transition is the negation of the guard on the other transition.

- The source of transition $t$ is not a super state, i.e., $\neg$ Super $(\operatorname{source}(t))$.

A state machine which satisfies our restrictions on a Rhapsody model is denoted by $S M_{r}$.

ComMA models A state machine in ComMA satisfies the following restrictions:

- States only have a name, no GUID. Names are unique, i.e., if $s_{1} \neq s_{2}$, then name $\left(s_{1}\right) \neq \operatorname{name}\left(s_{2}\right)$.

- There are no connector states.

- There are no sub state machines (and hence no super states), i.e., $S u b=\varnothing$.

Note that for readability we choose to use state names instead of GUIDs for the states in ComMA. To allow a straightforward translation from ComMA models to Dezyne we add one additional restriction on the ComMA models used in this paper:

- All transitions have an activation. 


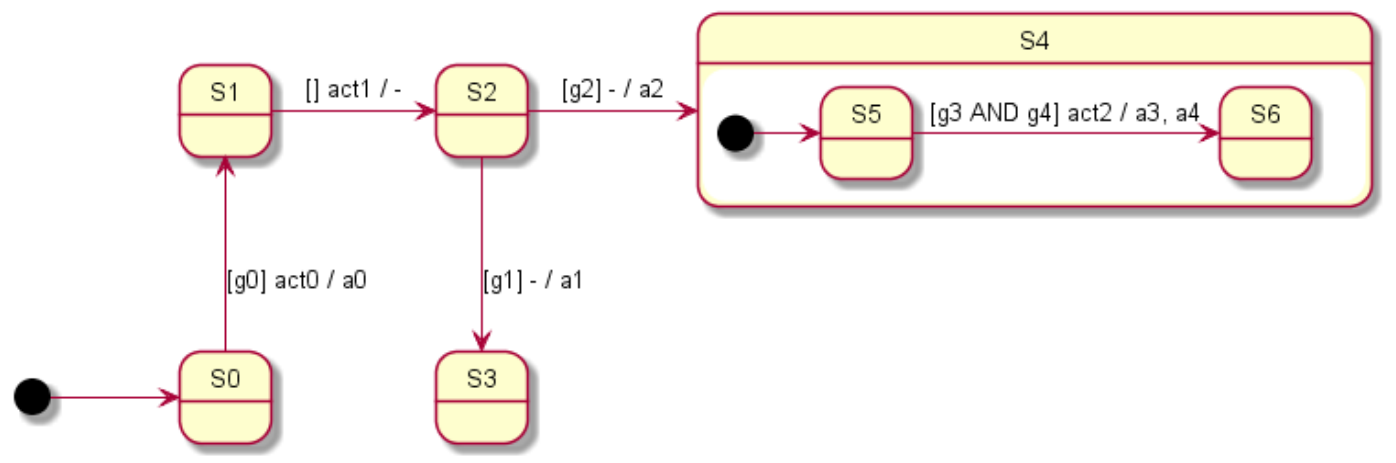

Figure 3. Example of a State Machine

A state machine which satisfies the restrictions above on a ComMA model is denoted by $S M_{c}$.

\subsection{From Rhapsody to ComMA}

We describe transformations to convert a Rhapsody into a ComMA model. The first four transformations are related to the restrictions on the ComMA models:

- Rename duplicate state names to allow removal of GUIDs.

- Remove hierarchy, i.e., flatten the sub state machines.

- Remove connector states.

- Add an activation to transitions without it.

Another transformation was added after our equivalence check showed a problem when we only used the above four transformations. By learning and comparing the Rhapsody and Dezyne state machines, we found out that the Rhapsody generated implementation is implicitly made input enabled by the Rhapsody run-time libraries. This behaviour is not described in the Rhapsody models. In the ComMA and Dezyne models all transitions have to be defined explicitly. This led to the fifth transformation:

- Make the state machine input enabled. Implicitly, all inputs are allowed in all states in Rhapsody models.

Details of these transformations are described in the next paragraphs. They are applied to a Rhapsody state machine $S M_{r}=<s_{d}, S, T, S u b>$.

Rename duplicate state names In the first transformation, duplicate states are renamed by the method RenameDuplicateNames. We use + for string concatenation and asString to convert a number to a string.

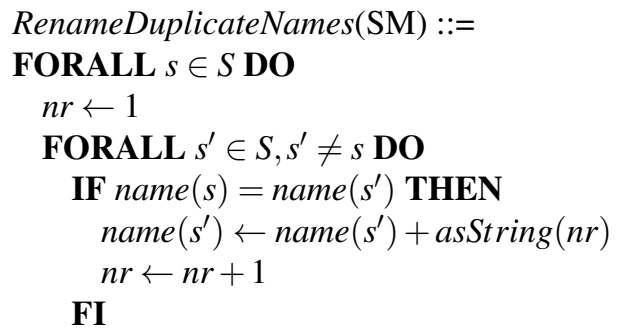

\section{OD \\ OD \\ RETURN $S M$}

Note that correctness of this transformation uses our assumption that state names are not post-fixed with a number.

Remove hierarchy Removing the hierarchy is done in a number of steps. To remove the sub state machines, we first remove transitions to super states as follows:

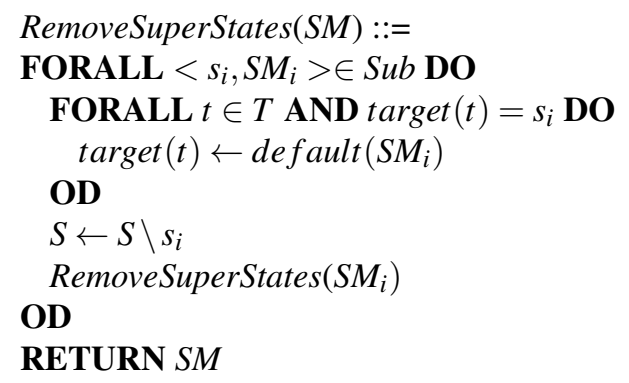

Note that the transformation is recursively applied to the sub state machines.

Next, all states and all transitions are collected by the following recursive definitions of $S_{t o t}(S M)$ and $T_{\text {tot }}$ for a state machine $S M$ :

- If $S u b=\emptyset$ then define $S_{t o t}(S M)=S$ and $T_{t o t}(S M)=T$.

- If $\left.S u b=\left\{<s_{1}, S M_{1}>, \ldots,<s_{n}, S M_{n}\right\rangle\right\}$ then define $S_{\text {tot }}(S M)=S \cup S_{\text {tot }}\left(S M_{1}\right) \cup \ldots \cup S_{\text {tot }}\left(S M_{n}\right)$ and $T_{\text {tot }}(S M)=T \cup T_{\text {tot }}\left(S M_{1}\right) \cup \ldots \cup T_{\text {tot }}\left(S M_{n}\right)$.

This is used in the following transformation:

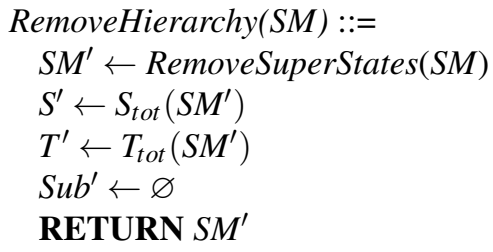

Remove connector states The connector states are removed using the transformation RemoveConnectorStates. Note that, by the restrictions on Rhapsody state machines, an outgoing transition of a connector state has no activation. 


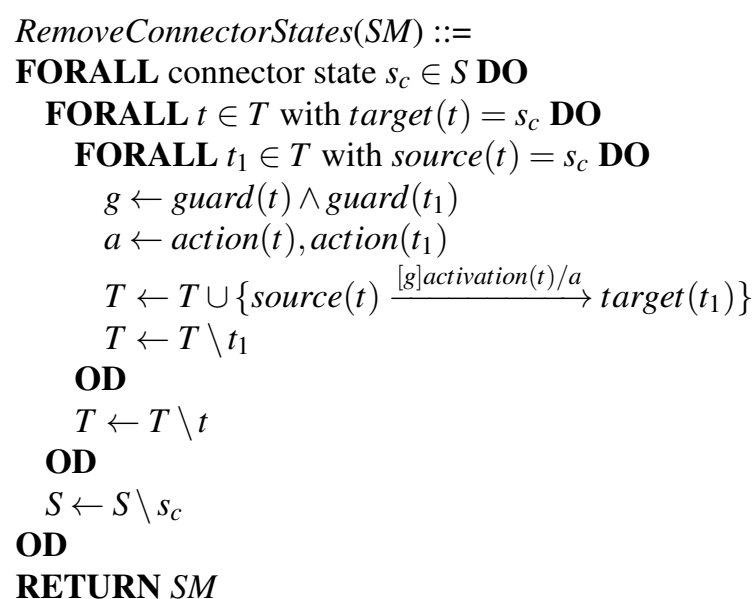

Make input enabled To ensure that in all states there is a transition for every activation, EnableInput adds self transitions with activations to states that are not input enabled. The reason for this transformation is explained in Section 5. EnableInput uses the following two definitions, for a set of transitions $T$ and a state $s$ :

- $\operatorname{Act}_{\text {tot }}(T)=\{\operatorname{activation}(t) \mid t \in T\}$, to collect all activations of the transitions in $T$

- $\operatorname{Act}(s, T)=\{\operatorname{activation}(t) \mid t \in T \wedge \operatorname{source}(t)=s\}$, to collect all activations used in state $s$

EnableInput $(S M)::=$

FORALL $s \in S$ DO

$A \leftarrow \operatorname{Act}_{\text {tot }}(T) \backslash A c t(s, T)$

FORALL act $\in A$ DO

$$
\text { OD }
$$$$
T \leftarrow T \cup\{s \stackrel{a c t /-}{\longrightarrow} s\}
$$

OD

\section{RETURN $S M$}

Add an activation to transitions To ensure that all transitions have an activation, every transition $t$ without an activation gets an activation event $\left(\right.$ event $\left._{E_{n}}\right)$, where $n$ is some unique number. All incoming transitions of source $(t)$ get an additional action $\left(\right.$ action $_{E_{n}}$ ) which places an event (event $E_{n}$ ) in the queue. This is formalized in transformation HandleActivation.

HandleActivation $(S M)::=$

$n r \leftarrow 1$

FORALL $t \in T$ without an activation DO

activation $(t) \leftarrow$ event $_{E_{n r}}$

FORALL $t^{\prime} \in T$ with target $\left(t^{\prime}\right)=\operatorname{source}(t)$ DO

OD

$\operatorname{action}\left(t^{\prime}\right) \leftarrow \operatorname{add}\left(\operatorname{action}\left(t^{\prime}\right), \operatorname{action}_{E_{n r}}\right)$

$n r \leftarrow n r+1$

OD

\section{RETURN $S M$}

Using these transformations, a Rhapsody state machine $S M_{r}$ can be converted to a ComMA model $S M_{c}$ as follows:
$S M_{1}=$ RenameDuplicateNames $\left(S M_{r}\right)$
$S M_{2}=$ RemoveHierarchy $\left(S M_{1}\right)$
$S M_{3}=$ RemoveConnectorStates $\left(S M_{2}\right)$
$S M_{4}=$ EnableInput $\left(S M_{3}\right)$
$S M_{c}=$ HandleActivation $\left(S M_{4}\right)$

Example After the described transformations, the state machine of Figure 3 is transformed into the state machine shown in Figure 4.

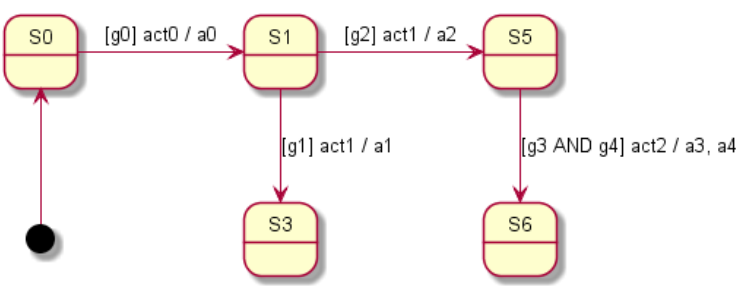

Figure 4. Result of Transforming the State Machine of Figure 3

\subsection{Generating ComMA Instances}

The mathematical description of the transformations in the previous subsection has been encoded in the generators of the RhapsodyDSL. The resulting ComMA model is defined by three files, as shown in Figure 5:

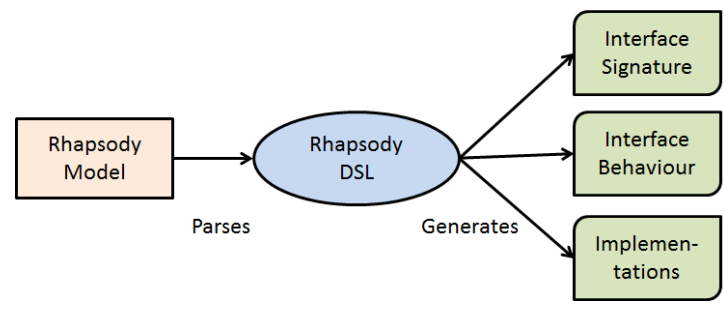

Figure 5. Generating Two ComMA Files and a Mapping File

- The .if file describes the signatures of the two interfaces defined: the first interface (IImpl) includes all activations, and the second interface (IUsed) includes all actions. Figure 6 shows the file for the example of Figure 4.

- The .sm file contains the state machine behaviour specification. Figure 7 contains part of the state machine of Figure 4; note that it provides interface IImpl and requires IUsed.

- The .mp file provides a mapping from the guards and actions to the source code implementation of these methods. Figure 8 provides an example of the mapping from actions and guards to their source code implementation. Here we assume that the transition $S_{0} \stackrel{[g 0] a c t 0 / a 0}{\longrightarrow} S_{1}$ of Figure 4 corresponds to the transition in the Rhapsody model fragment depicted in Figure 2. The mappings 
are separated by \#\#\#. Note that in the source code there are no lines starting with $=$ or \#\#\#.

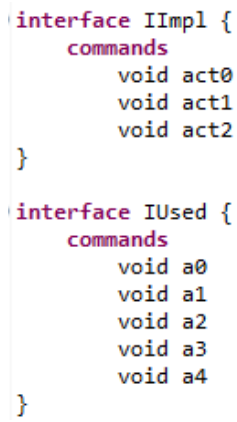

Figure 6. Example of a ComMA Interface File

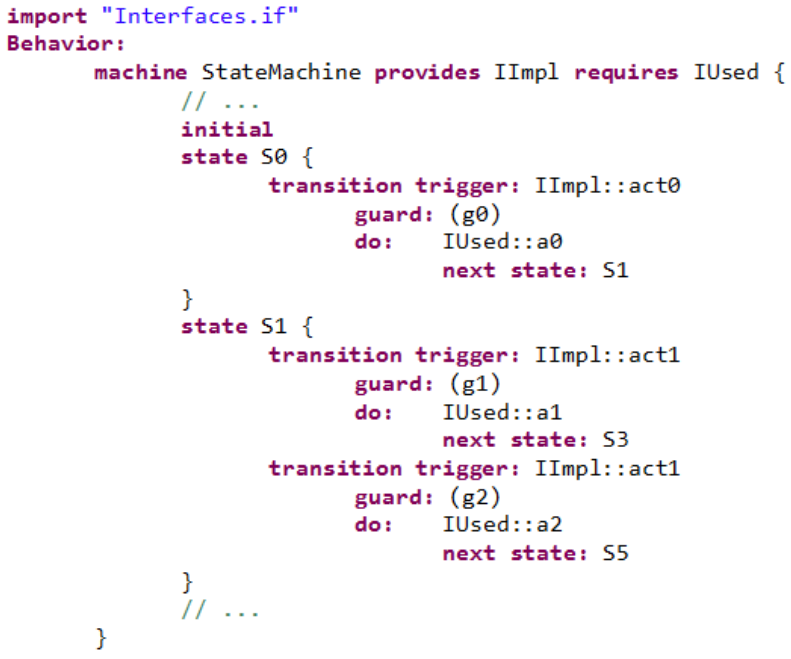

Figure 7. Example of a Specification of Interface Behaviour in ComMA

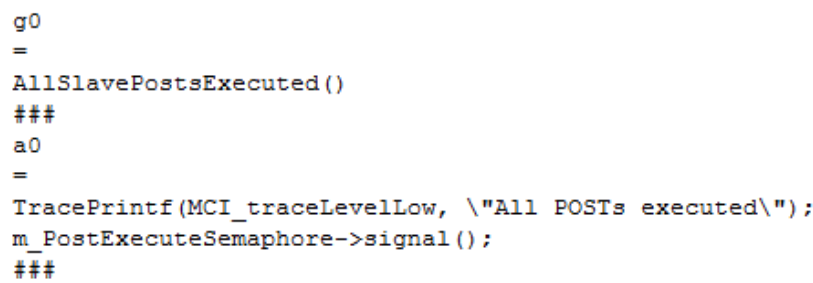

Figure 8. Example of a Mapping File

\subsection{From ComMA to Dezyne}

In the ComMA framework, we have defined a generator which transforms a ComMA model (i.e., an interface file and a state machine file) and a mapping file into a Dezyne model. The transformation is straightforward and shown here by an example.
The ComMA interfaces of Figure 6 are transformed into the Dezyne interfaces of Figure 9. Commands in the ComMA model are translated to methods in Dezyne. In Dezyne, the guards are part of the IUsed interface and return an enum value of type RVal which contains the values Ok and Nok.

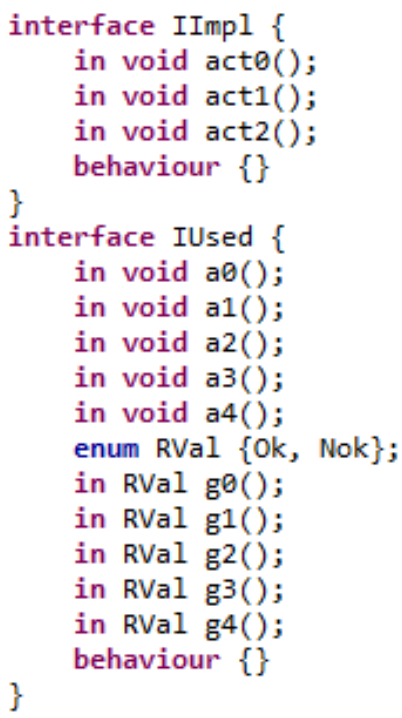

Figure 9. Dezyne Interfaces

The fragment of the ComMA state machine of Figure 7 is translated into the fragment of a Dezyne model shown in Figure 10. In the Dezyne model, the states are explicitly declared in an enum. The translation of the triggers is straightforward. Guards in the ComMA model are transformed into if-statements in the Dezyne model. Note that by our restrictions on a Rhapsody state machine, we have $g 2=\neg g 1$. In the Dezyne model this is generated as an else clause.

The mapping file is translated into glue code. For instance, for the mapping file of Figure 8, Dezyne glue code is generated as shown in Figure 11.

\section{Increasing Confidence in the Generated Code}

After model checking the Dezyne models, source code in $\mathrm{C}++$ is generated and integrated into a Visual Studio project. To get confidence that the state machines generated by Rhapsody and Dezyne behave the same, we first executed the existing regression test set. To further increase our confidence, we applied model learning, similar to [19].

The externally observable behaviour of an implementation can be learned using LearnLib [10], which is a model learner [25]. It generates a state machine in terms of an instance of the GraphViz's DOT-language [5]. Instances of this DOT language can be used to generate an instance of the mCRL2 language. The equivalence of two learned implementations can be checked using the equivalence checker from the mCRL2 tool set [2]. 


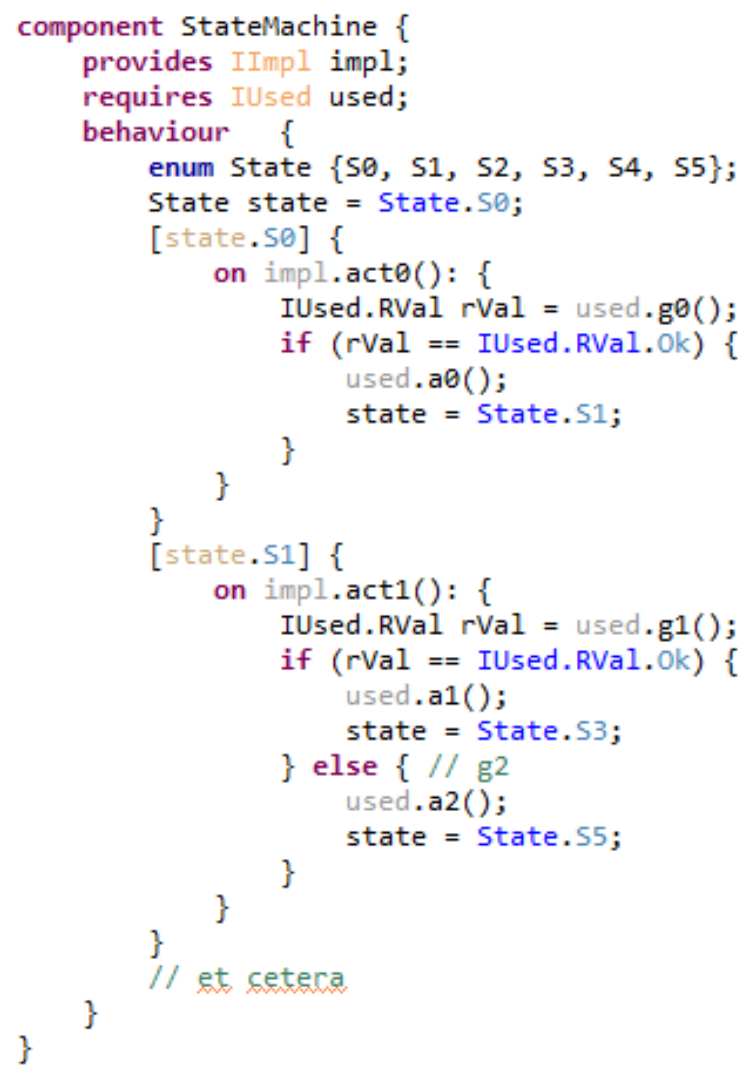

Figure 10. Dezyne Instance

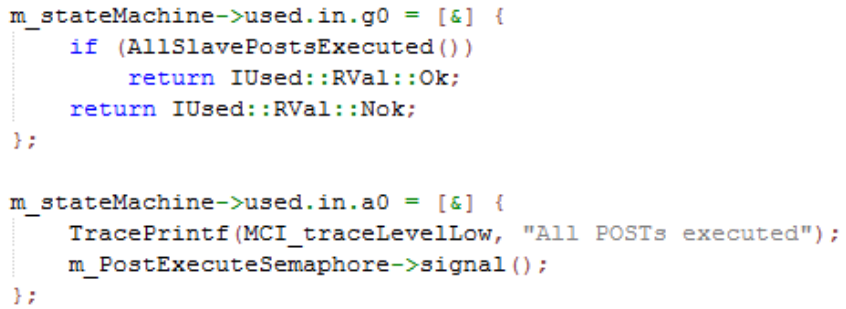

Figure 11. Dezyne Glue Code

Figure 12 depicts the place of model learning in our approach. The two versions of state machine code, generated by Rhapsody and Dezyne, are stimulated by all possible inputs and the resulting outputs are examined by the model learner. The two models that are the result of the learning phase are compared by the equivalence checker of the mCRL2 tool set.

With the learning approach, we found two errors in the Dezyne models that were not detected by the existing regression test set. The next two paragraphs describe the actions that were the results of these errors.

Make the model input enabled As mentioned in Section 4.2 we discovered the need for the EnableInput method which was not present in our first transformation from Rhapsody to
Dezyne. After adding this method, re-learning confirmed that both state machines are input enabled.

Add an activation to transitions The first HandleActivation method had a different implementation. Because every state with a transition without an activation had only one self-transition with an activation, these two transitions were combined to get a transition with an activation and the selftransition was removed. When we found out that Rhapsody models are input enabled, the HandleActivation method was modified according to the implementation described in Subsection 4.2.

After fixing these issues, re-learning confirmed that both state machines exhibit equivalent external behaviour. We did two learning experiments for learning the two state machines. The first state machine was learned with 14 stimuli and the second state machine was learned with 22 stimuli. Table 1 lists the final results of these two learning experiments with the number of states and the number of transitions found. Note that the number of transitions for Dezyne is larger than for Rhapsody because the state machines have to be made input enabled explicitly. The learned models have more states and transitions because model learning adds states and transitions to set the guards to true and false.

\section{Concluding Remarks}

In this paper, we presented an approach to use a RhapsodyDSL to transform Rhapsody models into Dezyne models. Model instances of the Rhapsody tool are instances of this DSL. The generator of the DSL transforms the models into model instances of the Dezyne modeling tool. We used ComMA as an intermediate language. To gain confidence in the transformation, model learning and equivalence checking have been applied.

Given the existence of powerful techniques such as Xtext and Xtend, this approach can be realized quickly and conveniently. In addition, it is rather easy and fast to create additional generators, for instance for visualizations. An alternative approach would be to write a script, for instance using Perl or Python, that does the parsing and transformation. We expect that this would consume more time and requires more expertise, e.g., concerning parsing.

We calculate the Return On Investment (ROI) for the presented DSL. First we compute the required investment for the model transformation approach. It took about 60 hours to understand the Rhapsody model structure, to create RhapsodyDSL and to add a Dezyne generator to ComMA. Model learning and equivalence checking, to increase the confidence, costed an additional 15 hours. Placing the two generated state machines into a Visual Studio project took another 25 hours. Hence, the total investment was approximately 100 hours.

A few months after this automatic transformation a second component with six state machines was manually rewritten. 


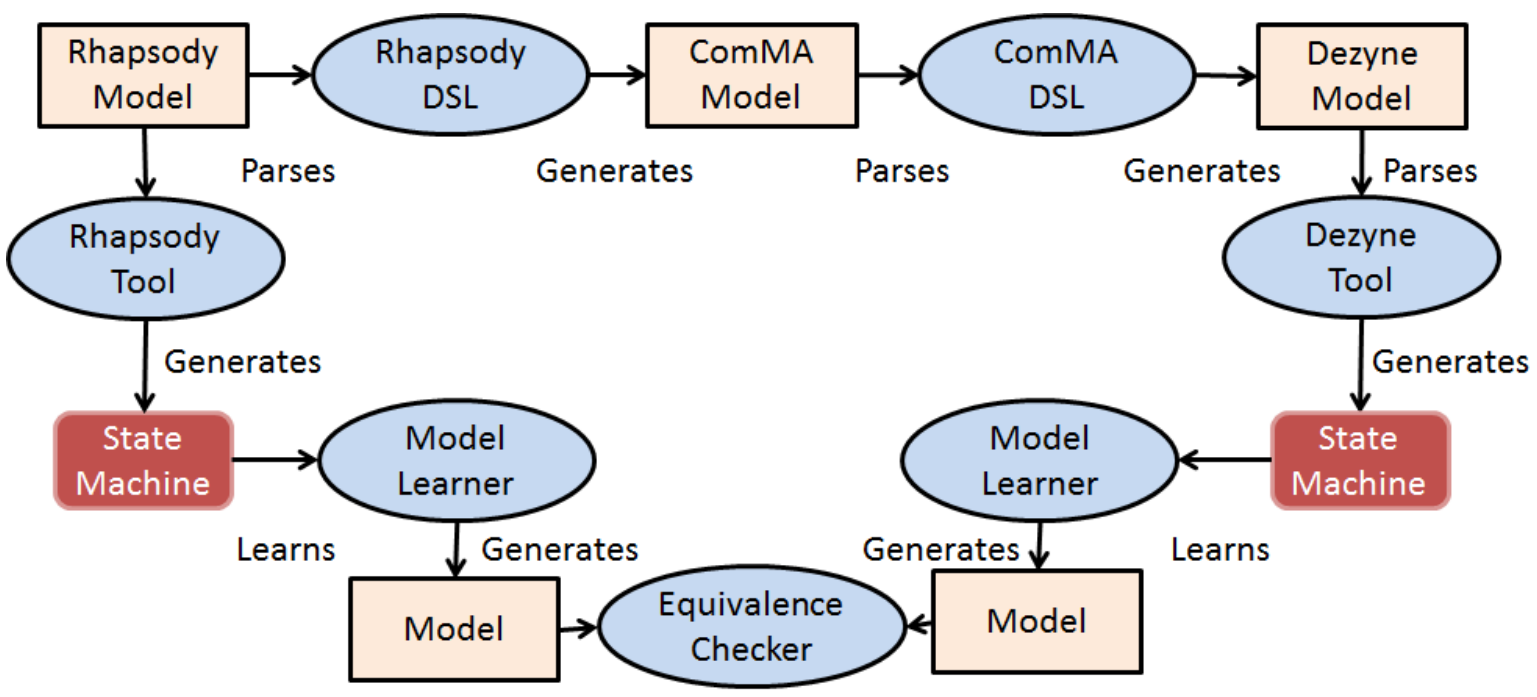

Figure 12. Learning Approach

\begin{tabular}{|l||l|l|l||l|l||l|l|l|}
\hline \multicolumn{1}{|c||}{ Experiment } & \multicolumn{4}{c||}{ Rhapsody } & \multicolumn{2}{c||}{ Dezyne } & \multicolumn{3}{c|}{ Learned } \\
\hline State machine & Super States & Sub States & Transitions & States & Transitions & States & Transitions & Stimuli \\
\hline 1 & 1 & 11 & 11 & 9 & 54 & 34 & 476 & 14 \\
\hline 2 & 2 & 10 & 17 & 6 & 30 & 58 & 1276 & 22 \\
\hline
\end{tabular}

Table 1. Results of Model Learning Applied to Two State Machines

The reason was that the state machines in this second component there exists a transition t such that (Super $(\operatorname{source}(t))$, a concept not supported by our approach. The first author of this paper was working on another project and did not have time to implement this new concept in the DSL. We estimate that it would take approximately 15 hours to add this new concept; 10 hours for RhapsodyDSL changes and 5 hours for learning the resulting state machines.

The manual transformation of the six state machines took 12 person weeks, which is $12 \times 36=432$ hours. To compare this to the DSL approach, we extrapolate this to the time required to implement all eight state machines: $(432 / 6) \times 8=$ 576. hours.

Next we calculate the time required to do the eight state machines with the DSL approach. In addition to the 100 hours for the first two state machines, this would require 15 hours to implement the new concept and $6 \times 12.5$ hours to integrate the state machines, leading to 190 hours.

This leads to the following Return On Investment of the DSL approach: $\mathrm{ROI}=$ (gain from investment - cost of investment $) /$ cost of investment $=(576-190) / 190 \approx 2$. This positive ROI value indicates that transforming the models automatically is preferred above manual transformations.

\section{References}

[1] L. Bettini. Implementing Domain-Specific Languages with Xtext and Xtend. Packt Publishing Ltd, 2013.

[2] S. Cranen, J. Groote, J. Keiren, F. Stappers, E. de Vink, W. Wesselink, and T. Willemse. An overview of the mCRL2 toolset and its recent advances. In Tools and Algorithms for the Construction and Analysis of Systems (TACAS), pages 199-213. Springer, 2013.

[3] K. Czarnecki and S. Helsen. Feature-based survey of model transformation approaches. IBM Systems Journal, 45(3):621-645, 2006.

[4] A. David, J. Deneux, and J. d'Orso. A formal semantics for UML statecharts. Technical Report 2003-010, Uppsala University, 2003. URL http://www.it.uu.se/research/reports/2003-010/.

[5] J. Ellson, E. Gansner, L. Koutsofios, S. C. North, and G. Woodhull. Graphviz-open source graph drawing tools. In International Symposium on Graph Drawing, pages 483-484. Springer, 2001.

[6] R. Eshuis. Reconciling statechart semantics. Sci. Comput. Program., 74(3):65-99, 2009.

[7] R. Grønmo and J. Oldevik. An empirical study of the UML model transformation tool (UMT). Proc. First Interoperability of Enterprise Software and Applications, Geneva, Switzerland, 2005.

[8] D. Harel and H. Kugler. The Rhapsody semantics of statecharts (or, on the executable core of the UML). In Integration of Software Specification Techniques for Application in Engineering, volume 3147 of LNCS, pages 325-354. Springer, Heidelberg, 2004.

[9] P. J. Hopcroft and G. H. Broadfoot. Combining the box structure development method and CSP for software development. Electron. Notes Theor. Comput. Sci., 128(6):127-144, 2005.

[10] F. Howar, M. Isberner, M. Merten, and B. Steffen. Learnlib tutorial: From finite automata to register interface programs. In T. Margaria, editor, ISoLA 2012, volume 7609 of LNCS, pages 587-590. Springer, Heidelberg, 2012. 
[11] IBM. Rational Rhapsody Developer, 2017. URL www.ibm.com/ software/products/en/ratirhapfami.

[12] F. Jouault, F. Allilaire, J. Bézivin, I. Kurtev, and P. Valduriez. ATL: a QVT-like transformation language. In Companion to the 21st ACM SIGPLAN symposium on Object-oriented programming systems, languages, and applications, pages 719-720. ACM, 2006.

[13] F. Jouault, F. Allilaire, J. Bézivin, and I. Kurtev. ATL: A model transformation tool. Science of computer programming, 72(1):31-39, 2008.

[14] I. Kurtev, M. Schuts, J. Hooman, and D.-J. Swagerman. Integrating interface modeling and analysis in an industrial setting. In Proceedings 5th International Conference on Model-Driven Engineering and Software Development, pages 345-352, 2017.

[15] T. Mens and P. Van Gorp. A taxonomy of model transformation. Electronic Notes in Theoretical Computer Science, 152:125-142, 2006.

[16] A. Mooij, G. Eggen, J. Hooman, and H. van Wezep. Cost-effective industrial software rejuvenation using domain-specific models. In International Conference on Theory and Practice of Model Transformations, pages 66-81. Springer, 2015.

[17] R. Pérez-Castillo, I. G.-R. De Guzman, and M. Piattini. Knowledge discovery metamodel-ISO/IEC 19506: A standard to modernize legacy systems. Computer Standards \& Interfaces, 33(6):519-532, 2011.

[18] M. Rosen, B. Lublinsky, K. Smith, and M. Balcer. Applied SOA: service-oriented architecture and design strategies. John Wiley \& Sons, 2012.

[19] M. Schuts, J. Hooman, and F. Vaandrager. Refactoring of legacy software using model learning and equivalence checking: an industrial experience report. In integrated Formal Methods, volume 9681 of LNCS, pages 311-325. Springer, 2016.

[20] G. Selim, S. Wang, J. Cordy, and J. Dingel. Model transformations for migrating legacy models: an industrial case study. In European Conference on Modelling Foundations and Applications, pages 90-101. Springer, 2012.

[21] G. M. Selim, S. Wang, J. R. Cordy, and J. Dingel. Model transformations for migrating legacy deployment models in the automotive industry. Software \& Systems Modeling, 14(1):365-381, 2015.

[22] S. Sendall and W. Kozaczynski. Model transformation: the heart and soul of model-driven software development. IEEE Software, 20(5): 42-45, 2003.

[23] U. Tikhonova, M. Manders, M. van den Brand, S. Andova, and T. Verhoeff. Applying model transformation and Event-B for specifying an industrial DSL. In MoDeVVa@ MoDELS, pages 41-50, 2013.

[24] L. Tratt. The MT model transformation language. In Proceedings of the 2006 ACM symposium on Applied computing, pages 1296-1303. ACM, 2006.

[25] F. Vaandrager. Model learning. Communications of the ACM, 60(2): 86-95, 2017.

[26] A. Van Deursen, E. Visser, and J. Warmer. Model-driven software evolution: A research agenda. Technical report, Delft University of Technology, Software Engineering Research Group, 2007.

[27] J. Wiegand et al. Eclipse: A platform for integrating development tools. IBM Systems Journal, 43(2):371-383, 2004. 